\title{
Analysis of Flow Characteristics in SCR Mixing Chambers of Different Shapes
}

\author{
Kyongjun Lee, Sechang Park \\ ${ }^{1}$ Korea Construction Equipment Technology Institute, Republic of Korea \\ 36, Sandan-ro, Gunsan-si, Jeollabuk-do, 54004 Republic of Korea \\ kjlee@koceti.re.kr; sc860425@koceti.re.kr
}

\section{Extended Abstract}

Most countries have recently applied strict emission regulations to non-road mobile machinery depending on the engine size for improving air quality. Most diesel vehicles are equipped with exhaust gas after-treatment systems to meet these emission regulations. Selective Catalytic Reduction (SCR), one of the exhaust gas after-treatment techniques, is used to efficiently remove the high concentration of NOx (nitrogen oxides) contained in exhaust gas. Urea water solution (UWS) is sprayed into the hot exhaust gas to obtain the ammonia which is the reactant for converting of NOx into nitrogen and water. To increase the efficiency of SCR, maximizing the mixing of UWS with exhaust gas is important. If mixing is not sufficient, the NOx reduction efficiency decreases and ammonia slip causes secondary pollution. It has been widely accepted that the thermal processes take place as the UWS is injected into hot exhaust gases [1,2]. We arranged a mixing chamber in front of the SCR to enhance mixing efficiency. The use of a mixing chamber is found to be beneficial for enhancing water vaporization and urea decomposition, and to improve the spatial distribution of the reductant. In this study, a three-dimensional, unsteady, multiphase flow analysis for mixing chambers was conducted using the ANSYS CFX 17.0 software to maximize the mixing of exhaust gases and UWS. A multi-phase numerical model has been developed to describe the key non-catalytic processes. The SCR region was approximated as porous media, and the injector nozzle considered in this study had six nozzle holes. Turbulence quantities were modeled using the RNG k- $\epsilon$ model for its improved capability over spray-generated turbulence. The influence of shape on mixing uniformity was investigated by calculating flow uniformity, meaning the spatial distribution of velocities and NH3 uniformity, which signifies the spatial distribution of ammonia. We considered three types of mixing chambers to ensure a high residence time, which is important for mixing exhaust gases and UWS. When the residence time is short, undecomposed urea can reach the catalyst face. Each type of mixing chamber was designed to increase the mixing efficiency by using swirl flow in the mixing chamber. A guide plate was incorporated into the mixing chamber to create swirl flow and a punched holes were arranged to ensure a high performance of mixing and transportation of mixed gases to the SCR inlet face. The optimal shape of the mixing chamber was selected by comparing pressure drop characteristics, flow uniformity, and NH3 uniformity among three shapes. The influence of the injection angle of the liquid urea injector on mixing uniformity was also investigated. The urea injection angle is critical for SCR simulation as the droplet size and their distribution play an important role in the evaporation and mixing processes. As a consequence, NOx conversion rate is strongly affected. This study is expected to provide important information that will be useful for designing SCR mixing chambers.

\section{Acknowledgements}

This Research is supported through the Ministry of Trade, Industry \& Energy (MOTIE) of the Republic of Korea (No.N052300003. 2016).

\section{References}

[1] M. Koebel, M. Elsender, M. Kleemann, "Urea-SCR: A Promising Technique to reduce NOx emissions from Automotive Diesel Engines," Catal. Today, vol. 59, pp. 335-345, 2000.

[2] S. D. Yim, S. J. Kim, J. H. Baik, I. S. Nam, Y. S. Mok, J. H. Lee, B. K. Cho, and S. H. Oh, "Decomposition of Urea into NH3 for the SCR Process," Ind. Eng. Chem. Res., vol. 43, pp. 4856-4863, 2004. 